\title{
Feministische Ökonomik als Gegenprogramm zur Standardökonomik: Kommentar zum Beitrag von Aysel Yollu-Tok und Fabiola Rodríguez Garzón
}

\author{
Notburga Ott
}

Online publiziert: 22. Februar 2019

(C) List-Gesellschaft e.V. 2019

Der Beitrag von Aysel Yollu-Tok und Fabiola Rodríguez Garzón bietet einen sehr guten Überblick über die verschiedenen Ansätze, die heute unter dem Terminus ,feministische Ökonomik“ subsumiert werden. Allesamt weisen sie auf unterschiedliche Aspekte weiblicher Lebensrealität hin, die in der Mainstream-Ökonomik vernachlässigt oder nur am Rande behandelt werden. Dass es sich dabei nicht um einen geschlossenen Theorieansatz, sondern um eine Vielzahl unterschiedlicher Kritikpunkte an der Mainstream-Ökonomie handelt, macht deutlich, wie vielschichtig und facettenreich die ausgeblendeten Lebensbereiche sind, die insgesamt mehr als nur einen vielleicht vernachlässigbaren blinden Fleck ausmachen. Insofern habe ich inhaltlich zu den verschiedenen Ansätzen wenig hinzuzufügen.

Allerdings möchte ich die Kategorisierung der Ansätze und die Abgrenzung zur Standardökonomik in Frage stellen - wenngleich sie so heutzutage auch international häufig zu finden ist. So werden gender economics und feminist economics als völlig unterschiedliche Ansätze beschrieben, auch wenn dies mit der deutschen Formulierung von feministischer Ökonomik im weiteren und engeren Sinn etwas abgeschwächt erscheint. Als gender economics werden dabei Ansätze innerhalb der Standardökonomik bezeichnet, die sich mit Frauen- und Geschlechterfragen beschäftigen und über das Instrumentarium der Standardökonomik nicht hinausreichen. Damit wird jedoch allen Ökonominnen, die mit den Instrumenten der Standardökonomik Frauenfragen bearbeiten, ein feministischer Anspruch abgesprochen, der als kritisches Hinterfragen der ,Theorien und Modelle der Standardökonomie und in einem feministischen Anliegen“" allein den feminist economics zugeordnet wird, die

This commentary article refers to the article available online at https://doi.org/10.1007/s41025-01900116-9.

N. Ott $(\bowtie)$

Ruhr-Universität Bochum, Bochum, Deutschland

E-Mail: notburga.ott@rub.de 
jedoch gleichzeitig als „Abgrenzung zur klassischen und neoklassischen Theorie“ definiert werden. Eine aus feministischer Perspektive grundsätzliche Kritik an der herrschenden Wirtschaftstheorie und alternative Modellbildung mit Instrumenten der Standardökonomik kann in dieser Abgrenzung nicht vorkommen.

Dies ist umso problematischer, als sich das als „Standardökonomik“ bezeichnete neoklassische Theoriegebäude auf den Stand der 60er bis 90er Jahre des letzten Jahrhunderts bezieht, das vom Mainstream heutiger Tage in dieser engen Form längst nicht mehr vertreten wird und vielfältige Erweiterungen - auch als Reaktion auf feministische und heterodoxe Kritik - erfahren hat. Von den Autorinnen werden jedoch ältere theoretische Ansätze mit empirischen Belegen aus der neueren Zeit konfrontiert, die sich erst jüngst so entwickelt haben. So widerlegen die von Frauen und Männern heute gleichermaßen erfolgenden Ausbildungsabschlüsse keineswegs die (Wenn-dann-)Aussage der Humankapitaltheorie, dass bei antizipierten Erwerbsunterbrechungen geringere Humankapitalinvestitionen getätigt werden - diese ist heute schlichtweg empirisch nicht mehr relevant, da sich die Rahmenbedingungen und damit die Erwartungen geändert haben. Die eigentlich spannende Frage ist, warum wir heute andere empirische Befunde vorfinden als in den 60er und 70er Jahren, wo entsprechende Erwartungshaltungen sehr wohl die Bildungsentscheidungen der Mädchen und Jungen und deren Eltern beeinflusst haben. Hierzu kann die Standardökonomik entscheidende Hinweise geben, denn der Homo Oeconomicus existiert keineswegs in einem ,institutionsfreien Raum“, wie die Autorinnen behaupten, sondern die Rahmenbedingungen, die durch Institutionen gesetzt werden, fallen unter die vom Homo Oeconomicus zu berücksichtigenden Restriktionen, die er in Interaktion mit anderen Wirtschaftssubjekten selbst (mit-)gestaltet (vgl. Ott 2001). Allerdings wird dieses Erklärungspotential von den Neoklassikern der 70er Jahre und insbesondere den Vertretern der sogenannten New Home Economics nicht hinreichend genutzt, da sie - entgegen dem theoretischen Modell - statt auf Rahmenbedingungen auf naturgegebene Präferenzen rekurrieren, woraus dann die zu Recht kritisierte zirkuläre Argumentation folgt. Werden die strukturellen Rahmenbedingungen und deren pfadabhängige Entwicklung jedoch explizit formuliert, dann zeigt sich, dass nicht die Argumentation zirkulär ist, sondern die gesellschaftlichen Bedingungen einen solchen ,Teufelskreis ökonomischer Rationalität“ erzeugen (vgl. Ott 1999). Es ist somit nicht die neoklassische Theorie, die die blinden Flecken erzeugt, sondern die vorurteilsbehaftete oder sogar ideologische Blindheit der jeweiligen Ökonomen, die ihr eigenes Instrumentarium nicht konsequent anwenden. In dynamischer Betrachtung können dann auch die Entwicklung und Veränderung sowohl privater Wirtschafts- und Lebensgemeinschaften als auch gesellschaftlicher Rechtsordnungen und kollektiver Sicherungssysteme als (zumindest teilweise) Folge der wirtschaftlichen Entwicklung rekonstruiert und deren situationsbezogene (temporäre) Vorteilhaftigkeit wie auch ihre Verteilungswirkungen analysiert werden (vgl. Ott 2009). Allerdings genügt hierzu nicht mehr der enge neoklassische Ansatz der 60er Jahre, sondern es werden Argumentationsmuster neuerer ökonomischer Teilgebiete insbesondere der Institutionenökonomik genutzt, die eine Erweiterung des neoklassischen Ansatzes darstellen und heute durchaus Teil der Mainstream-Ökonomik sind. 
Auch wenn daher das Instrumentarium der Standardökonomik deutlich mehr zur Analyse der Lebenswirklichkeit von Frauen beitragen kann als manche Kritikerinnen zugestehen, ist dennoch eine Vielzahl von relevanten Aspekten mit dem Ansatz der Standardökonomik nicht fassbar. Macht, Gewalt, Emotionen wie Liebe, Hass und Neid entziehen sich der Analyse mit dem standardökonomischen Instrumentarium, bestimmen aber die Lebenswirklichkeit in so gravierender Weise, dass ihr Ignorieren - bewusst oder unbewusst - zu eklatanten Fehlschlüssen führen muss. Feministische Ansätze, die dies zu überwinden versuchen, sind daher berechtigt und unbedingt notwendig. In ökonomischen Ansätzen der Standardökonomik sollten diese Aspekte - soweit dies überhaupt geht - als Randbedingungen mit betrachtet werden, was aber leider von vielen Mainstream-Ökonomen unterlassen oder in unfruchtbarer und tautologischer Weise eingeführt wird (Ott 2001). Generell ist es aber notwendig, die Grenzen des (standard-)ökonomischen Ansatzes zu kennen und sich diskursiv mit alternativen theoretischen Ansätzen und den Erkenntnissen anderer Disziplinen auseinander zu setzen, ohne die Erklärungskraft des eigenen Ansatzes völlig in Frage zu stellen. Ich persönlich bin nicht der Meinung, dass alle ausgeblendeten Aspekte in die ökonomische Theorie integriert werden müssen, sondern ich schätze die wissenschaftliche Arbeitsteilung unterschiedlicher Disziplinen und Ansätze sehr, weil sie die Aussage des jeweils betrachteten Zusammenhangs, der immer nur ein Ausschnitt der Lebensrealität sein kann, schärfen. Um daraus aber politische Folgerungen ziehen zu können, ist der Diskurs mit den Erkenntnissen anderer Ansätze jedoch unverzichtbar.

Abschließend lohnt es sich, vor dem Hintergrund der wechselseitigen Beeinflussung von konkurrierenden wissenschaftlichen Ansätzen einen Blick auf die erkenntnistheoretische Verortung der Entwicklung feministischer Ökonomik zu werfen. Auch wenn diese in einem Überblicksartikel nicht erwartet werden kann, werden die knappen Ausführungen dazu der Entwicklung der verschiedenen Positionen im letzten Jahrhundert - sowohl innerhalb der feministischen Bewegung, innerhalb der wirtschaftswissenschaftlichen Community als auch in der Gesellschaft - nicht ganz gerecht. Der Fokus der feministischen Ökonomik war keineswegs von Anfang an auf den Arbeitsmarkt gerichtet. Die Pionierinnen beschäftigten sich vor allem mit der Frage nach dem Verhältnis und Stellenwert von bezahlter und unbezahlter Arbeit, von marktmäßiger Arbeit und Hausarbeit und den daraus folgenden Abhängigkeitsverhältnissen. Und die feministische Ökonomik der zweiten Frauenbewegung verstand sich nicht originär als Gegenmodell zur Standardökonomik, sondern suchte in der sogenannten „Hausarbeitsdebatte“ die Konfrontation mit der marxistischen Ökonomik, in der die Spezifität von Hausarbeit ebenfalls nicht vorkommt, sondern nur als unbezahlte Arbeit der Frauen dem Kapitalismus angelastet wurde. Die vorrangige Beschäftigung mit dem Arbeitsmarkt begann in den 80er Jahren als Folge verschiedener Entwicklungen: die steigende Erwerbsbeteiligung von Frauen, die durch die Frauenbewegung in die Öffentlichkeit geratene Sichtbarkeit der Diskriminierung am Arbeitsmarkt, sowie die zunehmende Verfügbarkeit von empirischen Arbeitsmarktdaten, wodurch die unbezahlte Hausarbeit in den Schatten der wissenschaftlichen Forschung geriet. Die jüngere Fokussierung auf Care-Arbeit schließt jedoch nicht direkt an die alte Frage nach unbezahlter Hausarbeit an, die neben Reproduktions- und Sorgearbeit noch umfassend den Aspekt der materiellen 
und immateriellen Eigenproduktion beinhaltete. Da diese in den vergangenen Jahrzehnten erheblich zurückgegangen ist und durch Marktsubstitute ersetzt wurde (Ott 2009), ist allerdings heute die Care-Arbeit durchaus der Kern des Problems. Gerade dieser Aspekt der jüngeren wirtschaftlichen Entwicklung ist es wert, genauer betrachtet zu werden, um die moderne „Abhängigkeitsfalle“ von Frauen im Vergleich $\mathrm{zu}$ früheren Gesellschaftsmodellen, auf denen unsere Sozialsysteme immer noch basieren, angemessener einordnen zu können.

Um dem feministischen Anspruch gerecht zu werden, nicht nur Wissen zu generieren, sondern ,,bestehende Politiken zu analysieren und parteiisch Ziele zu formulieren“ und in diesem Sinne ,,verändernde und intervenierende Praxis“ zu verfolgen, ist der Diskurs der verschiedenen Strömungen der feministischen Ökonomik unabdingbar. Der Androzentrismus der herrschenden Wirtschaftstheorie kann wohl nur durch die gemeinsame Kritik von innen wie von außen überwunden werden.

\section{Literatur}

Ott, N. (1999). The Economics of Gender - Der neoklassische Ansatz zur Erklärung des Geschlechterverhältnisses. In B. Dausien, M. Herrmann, Oechsle, Mechtild, C. Schmerl \& M. Stein-Hilbers (Hrsg.), Erkenntnisprojekt Geschlecht - die Geschlechterperspektive in den Einzelwissenschaften (S. 167-196). Opladen: Leske + Budrich.

Ott, N. (2001). Der Ordnungsbezug des Ökonomischen Imperialismus - Das Beispiel der Familienökonomik. In H. Leipold \& I. Pies (Hrsg.), Ordnungstheorie und Ordnungspolitik - Konzeptionen und Entwicklungsperspektiven. Schriften zu Ordnungsfragen der Wirtschaft, (Bd. 64, S. 171-196). Stuttgart: Lucius\&Lucius.

Ott, N. (2009). Wie sichert man die Zukunft der Familie? In N. Goldschmidt (Hrsg.), Generationengerechtigkeit. Reihe: Untersuchungen zur Ordnungstheorie und Ordnungspolitik. (S. 47-69). Tübingen: J.C.B. Mohr. 